\title{
microRNA signals cell fate
}

In signalling networks, a positive-feedback loop can form when a weak or transient signal enhances expression of components of its pathway to amplify or stabilize a particular cellular response. Li and Carthew (Cell 123, 1267; 2005) recently described an alternative, double-negative feedback mechanism in the fly eye that involves interplay of a transcription factor with a microRNA (miRNA) to drive developmental decisions.

miRNAs are members of a family of non-protein-coding RNAs that inhibit target $m R N A s$ by binding to complementary sequences within the message, thus preventing synthesis of the protein product. The regulation of miRNAs, and their specific cellular roles, remains largely obscure. When searching for the function of miRNAs in Drosophila eye development, Li and Carthew identified active $m i R-7$ in differentiating photoreceptors. Forced expression of $m i R-7$ resulted in ectopic formation of photoreceptor neurons, which resembled the phenotype observed when the transcriptional repressor Yan is lost.

In the Drosophila eye, Yan normally prevents progenitors from spontaneously differentiating. Differentiation is initiated through EGFRERK signalling, which promotes the degradation of Yan. The authors showed that $m i R-7$ misexpression reduces the levels of endogenous Yan (or of a Yan reporter transgene), whereas Yan was no longer repressed in a $m i R-7$ null background. Furthermore, a mutant form of Yan that is not controlled by ERK-dependent degradation, was also negatively regulated by miR-7; conversely, Yan's inhibitory effect on photoreceptor differentiation was enhanced in a miR-7 null background.

If $m i R-7$ directly downregulates Yan expression during differentiation, what keeps $m i R-7$ levels in check? Is $m i R-7$ function also under the control of EGFR? Li and Carthew observed that forced expression of a constitutively active mutant of EGFR in progenitor cells resulted

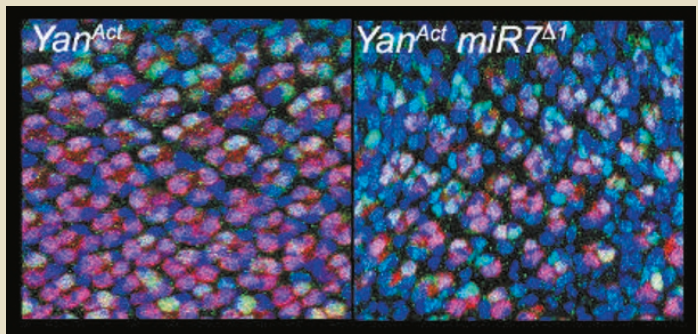

miR-7 promotes photoreceptor development. Expression of ERKindependent Yan in retinal photoreceptor neurons (red) results in the failure of some cells to differentiate into photoreceptors (blue; left panel). This phenotype is exacerbated when miR-7 is also missing (right panel).

in $m i R-7$ upregulation and that, interestingly, $m i R-7$ was ectopically expressed in yan loss-of-function mutants. The authors showed that Yan directly represses $m i R-7$ expression in progenitor cells and when Yan is degraded (through EGFR-ERK signalling) it can no longer repress the transcription factor Pointed-P1 - an activator of $m i R-7$ expression in photoreceptors.

The work of Li and Carthew describes a reciprocal negative-feedback pathway between a protein and a miRNA. During eye development, this regulatory loop ensures the mutually exclusive expression of Yan and miR-7, in progenitor cells and photoreceptors, respectively. EGFR-ERK signalling switches the expression of these genes by inducing the degradation of Yan, thereby relieving the inhibition of miR-7. Whether or not other miRNAs are involved in such intricate relations with transcription factors during cell-fate specification has yet to be determined.

MYRTO RAFTOPOULOU 